\title{
Inhibition of epidural fibrosis after microendoscopic discectomy with topical application of mitomycin C: a randomized, controlled, double-blind trial
}

\author{
Clinical article
}

\author{
*Lei Liu, M.D., ${ }^{1}$ Tao Sui, M.D., ${ }^{2}$ Xin Hong, M.D., ${ }^{1}$ ХiaOtao Wu, M.D., Ph.D., ${ }^{1}$ \\ and Xiaojian Cao, M.D., Ph.D. ${ }^{2}$ \\ ${ }^{1}$ Department of Orthopedics, the Affiliated ZhongDa Hospital of Southeast University; and ${ }^{2}$ Department of \\ Orthopedics, the First Affiliated Hospital of Nanjing Medical University, Nanjing, JiangSu Province, China
}

\begin{abstract}
Object. The authors conducted a study to evaluate the effects and the safety of locally applied mitomycin C (MMC) on epidural fibrosis after microendoscopic discectomy (MED).

Methods. Seventy-five patients undergoing single-level unilateral MED for lumbar disc herniation were randomly assigned to receive cotton wool impregnated with either $0.5 \mathrm{mg} / \mathrm{ml} \mathrm{MMC} \mathrm{or} \mathrm{saline} \mathrm{applied} \mathrm{at} \mathrm{the} \mathrm{site} \mathrm{of} \mathrm{discec-}$ tomy for 5 minutes. Outcome measures included degrees of pain severity, functional disability, physical symptoms, and quantitative evaluation of postoperative epidural fibrosis shown on follow-up lumbar contrast-enhanced MRI.

Results. Sixty-two patients completed the follow-up. Neither serious drug adverse effects nor clinically significant laboratory adverse effects were observed. Patients in both groups showed similar clinical recoveries postoperatively. A statistically significant difference $(p<0.05)$ between the 2 treatments was shown in a quantitative evaluation of postoperative MRI-documented epidural fibrosis in the MMC group and the saline group using a modified grading system. The mean cross-sectional areas of epidural fibrosis were 7.32-70.06 $\mathrm{mm}^{2}$ in the MMC group and 22.94-90.48 $\mathrm{mm}^{2}$ in the saline group. The epidural fibrosis index ranged from 0.0296 to 0.3267 in the MMC group and from 0.1191 to 0.3483 in the saline group. A significant difference was also observed using the Ross grading system to evaluate postoperative MR images.

Conclusions. Although no benefit was observed clinically, the authors observed a notable reduction of epidural fibrosis after MED radiologically, with $0.5 \mathrm{mg} / \mathrm{ml} \mathrm{MMC} \mathrm{locally} \mathrm{applied} \mathrm{and} \mathrm{no} \mathrm{clinical} \mathrm{side} \mathrm{effects.} \mathrm{Clinical} \mathrm{trial}$ registration no.: ChiCTR-TRC-10001079 (http://www.chictr.org/cn/proj/show.aspx?proj=326).

(http://thejns.org/doi/abs/10.3171/2013.1.SPINE12564)
\end{abstract}

\section{KEY WORDS • epidural fibrosis • microendoscopic discectomy $\quad$ mitomycin C disc herniation $\bullet$ lumbar}

E PIDURal fibrosis has often been described as a main factor leading to undesirable postoperative symptoms and pain recurrence and therefore poorer outcome after the spinal surgeries. Furthermore, epidural adhesions greatly increased the risk of nerve root injury and dural tears, which makes the second exposure of the same operative area technically difficult and dangerous. . $, 8,11,14^{2}$ As a result, multiple surgical techniques have been proposed and various antiadhesion agents have been tested to minimize postoperative scarring. $5,8,9,12,13$

\footnotetext{
Abbreviations used in this paper: $\mathrm{CSA}=$ cross-sectional area; LSOQ = Lumbar Spine Outcomes Questionnaire; MED = microendoscopic discectomy; $\mathrm{MMC}=$ mitomycin $\mathrm{C}$.

* Drs. Liu and Sui contributed equally to this work.
}

Mitomycin C, used as a suppressive agent of fibroblast proliferation, has been shown to significantly reduce epidural fibrosis in well-designed animal models. A prospective randomized clinical study was performed to evaluate the effects of preventing epidural fibrosis after lumbar microdiscectomy, but it ended with frustrating results. ${ }^{4}$

The present study was designed to evaluate the effect of MMC on inhibiting epidural fibrosis after lumbar MED and to establish the presence of side effects; the procedures were conducted by the same surgeon using the same technique in 2 groups of patients. In one group, $0.5 \mathrm{mg} / \mathrm{ml} \mathrm{MMC} \mathrm{was} \mathrm{applied} \mathrm{at} \mathrm{the} \mathrm{site} \mathrm{of} \mathrm{the} \mathrm{discec-}$ tomy for 5 minutes, and in the other group, the control group, physiological saline was applied in the same way. 
We used a modified grading system to evaluate the extent of epidural fibrosis depicted on follow-up MR images.

\section{Methods}

\section{Study Population}

Seventy-five patients undergoing single-level unilateral MED for lumbar disc herniation were enrolled consecutively for study between March 2010 and December 2010 from regular clinical practice in a single institution (Department of Orthopedics, Affiliated ZhongDa Hospital of Southeast University). All patients fulfilled the following study inclusion criteria: clear clinical signs consistent with the diagnosis of lumbar radiculopathy, evidence of lumbar disc herniation on MRI or CT, and consistency between symptoms, neurological examination, and radiological findings. Exclusion criteria were age greater than 60 years or less than 20 years; multiple levels of lumbar disc herniation, pyogenic discitis, or other infections; disc herniation associated with spondylolisthesis; spinal canal stenosis; any prior lumbar spine surgeries; local or general infection; allergic reaction; peroral administration of aspirin or other nonsteroidal antiinflammatory drugs within the 7 days preceding the surgery; active psychiatric disease; or severe heart or respiratory failure. Patients were also excluded if the dura mater was injured intraoperatively.

\section{Study Design}

The study was a prospective, randomized, controlled, double-blind clinical trial. The study protocol was approved by the ethics committee of the First Affiliated Hospital of Nanjing Medical University and was registered on the Chinese Clinical Trial Registry (ChiCTRTRC-10001079). The study was performed on the basis of the Consolidated Standards of Reporting Trials (also known as CONSORT). Written informed consent was obtained from all the participating patients. Patients enrolled in the study were allocated randomly, according to computer-generated random numbers sealed in opaque envelopes with consecutive numbering, into 1 of the 2 groups by an orthopedist who was not involved in the study. At the end of the MED procedure, the same orthopedist responsible for establishing the patient's assignment then instructed a nurse, who was blinded to patient allocation number, to dispense either the MMC (Kyowa Hakko Kirin Co., Ltd.) or physiological saline.

\section{Blinding}

Patients and those administering the interventions were all blinded to group assignment. The blinding was ensured by mixing the patients with other patients receiving routine treatment. The surgeon performing the procedure was also blinded to the group assignment. The results of 6-month follow-up were tabulated by a statistician not participating in provision of patient care. The unblinded results were not disclosed to the surgeon, the other participants, or patients.

\section{Surgical Procedures}

All MED procedures were performed by the same se- nior surgeon (X.W.) using the METRx system (Medtronic Sofamor Danek) after epidural induction of anesthesia. Flavectomy was performed via a standard longitudinal splitting approach, and laminotomy was performed using an upward-angled curette and a Kerrison punch to expose the dura and traversing nerve roots. Subsequently, nerve root retraction and discectomy were conducted to remove herniated disc material with a pituitary rongeur. ${ }^{12,17}$ After strict hemostasis, a piece of cotton wool $(1 \times 5 \mathrm{~cm})$ impregnated with $0.5 \mathrm{mg} / \mathrm{ml} \mathrm{MMC} \mathrm{was} \mathrm{applied} \mathrm{around} \mathrm{the}$ nerve, including the anterior, lateral, and posterior space in the MMC treatment group. Five minutes later, the MMC-soaked cotton wool was removed, and the laminectomy sites were irrigated immediately with saline solution to eliminate the surplus MMC. In patients in the control group a piece of cotton wool impregnated with physiological saline was applied locally for 5 minutes in the same way. Finally the cotton fiber, bone chips, and disc fragments were cleared away, and the lumbodorsal fascia and subcutaneous tissue were closed with absorbable sutures.

\section{Outcome Measures}

Clinical Follow-Up. Preoperative and postoperative examinations were performed by the same orthopedists (C.M. and X.W.) in a blinded manner. The postoperative clinical follow-up examinations were performed during the 1st week, and then 3 and 6 months postoperatively. The $\mathrm{LSOQ}^{3}$ and neurological examinations were used to evaluate the clinical outcomes. For each patient and for each evaluation period, scores on 4 composite measures were derived from the responses to the LSOQ: a lumbar back pain severity score, leg pain severity score, physical symptom and radiculopathy score, and functional disability and activity-related pain score. A different point scale was used to indicate the severity of the 4 composite measures. Additionally, lower-extremity weakness was evaluated using a motor scale, with scores ranging from 0 (no motor weakness) to 5 (complete motor functional loss).

Radiological Follow-Up. The MRI examinations were performed 3 months postoperatively following a standard protocol. All patients were in the supine psoasrelaxed position. ${ }^{6}$ A spinal surface coil (FOV $25 \mathrm{~cm}$ ) was used at a field strength of $1.5 \mathrm{~T}$ (Siemens); the slice thickness was $4 \mathrm{~mm}$; and matrices applied were $256 \times 256$ for the sagittal and axial sections. In all patients, sagittal T1weighted spin echo images (TR/TE 300/22 msec), sagittal T2-weighted images (TR/TE 3900/150 msec), and axial T1-weighted images (TR/TE 300/22 msec) were obtained within 10 minutes after intravenous administration of $0.1 \mathrm{mmol} / \mathrm{kg}$ of Gd-DTPA (Magnevist, Bayer Schering Pharma). Axial images were obtained in a gap-and-fill fashion to allow complete coverage of the surgical site and were contiguous (that is, not only the operative disc level). Axial images covered 2 levels above the central axial slice of the operative intervertebral disc to 2 levels below. For the purpose of evaluating epidural fibrosis, we used only the axial T1-weighted images with and without contrast enhancement.

All MR images were reviewed independently by 2 


\section{Inhibition of epidural fibrosis by mitomycin $\mathrm{C}$}

fellowship-trained orthopedists (X.H. and J.W.) blinded to the operative and clinical findings. The Ross grading system ${ }^{15}$ and a modified grading system were used during the evaluating procedure.

The Ross Grading System. The 5 contiguous axial slices centered around the surgically treated intervertebral disc were observed. Each of the 5 levels was further subdivided into 4 quadrants defined by perpendicular lines drawn from the central aspect of the thecal sac. For each quadrant at each imaging slice encompassing the operative level, the amount of epidural fibrosis was graded on the following scale of 0-4: Grade 0, no scar or trace of a scar; $1>0 \%$ to $\leq 25 \%$ of the quadrant filled with scar; $2,>25 \%$ to $\leq 50 \%$ of the quadrant filled with scar; $3,>50 \%$ to $\leq 75 \%$ of the quadrant filled with scar; and $4,>75 \%$ to $\leq 100 \%$ of the quadrant being filled with scar. Therefore, a total of 20 scores were obtained for each operative level encompassing the 5 imaging slices. The 2 orthopedists independently evaluated 1240 quadrants of 5 slices in all 62 patients. The results were further compared and analyzed.

Modified Grading System. The evaluation of the MR images detailed the location of the epidural fibrosis on 5 contiguous axial slices centered on the intervertebral disc (Fig. 1). The 5 slices included those of the lateral recesses, exiting root, anterior epidural space, dural sac, and posterior epidural space. Differing from the grading system presented by Ross et al., ${ }^{15}$ the amount of epidural scarring in the vertebral canal space was given a score of $0-10$ on each slice. In our modified grading system, a score of 0 indicates no scar; score of 1 is a slice with $1 \%-10 \%$ scar filling; score of 2, 11\%-20\%; score of 3, 21\%-30\%; score of $4,31 \%-40 \%$; score of $5,41 \%-50 \%$; score of $6,51 \%-$ $60 \%$; score of $7,61 \%-70 \%$; score $8,71 \%-80 \%$; score of 9 ,

\begin{tabular}{|c|c|c|c|}
\hline & $\begin{array}{l}\text { Schematic } \\
\text { representation }\end{array}$ & Image & Explanation \\
\hline & & & $\begin{array}{l}\text { The MR slices were positioned parallel to the endplate of } \\
\text { the vertebra above/below the operative disc. The slice } \\
\text { thickness was } 4 \mathrm{~mm} \text {. }\end{array}$ \\
\hline Slice 1 & & & $\begin{array}{l}\text { The border of the vertebral canal is defined by the artifi- } \\
\text { cial boundary line in the schematic diagram. The image } \\
\text { shows no epidural fibrosis in the epidural space. }\end{array}$ \\
\hline Slice 2 & & & No epidural fibrosis in the epidural space. \\
\hline Slice 3 & & & $\begin{array}{l}\text { The central axial slice of the operative intervertebral disc. } \\
\text { Intervertebral disc shows hypointensity. }\end{array}$ \\
\hline Slice 4 & & & $\begin{array}{l}\text { The vertebral canal is entirely surrounded by the poste- } \\
\text { rior aspect of the vertebral body anteriorly and by the } \\
\text { vertebral pedicles and laminae posterolaterally. Epidural } \\
\text { fibrosis shows hypointensity. }\end{array}$ \\
\hline Slice 5 & & & $\begin{array}{l}\text { The left lamina was partly absent. A subcutaneous op- } \\
\text { erative channel, lateral to the spinous process, shows hy- } \\
\text { pointensity. }\end{array}$ \\
\hline
\end{tabular}

FIG. 1. Schematic representations and the corresponding MR images detailing the location of the epidural fibrosis. 
$81 \%-90 \%$; and score $10,91 \%-100 \%$ scar filling the space. Additionally, the CSAs of vertebral canal $\left(\mathrm{CSA}_{\mathrm{vc}}\right)$, dural sac $\left(\mathrm{CSA}_{\mathrm{ds}}\right)$, and nerve roots $\left(\mathrm{CSA}_{\mathrm{nr}}\right)$ were measured by using a standard measurement program for MR images. Axial images were cross-referenced with sagittal localizing images to ensure accuracy. During measurement, the partitions of the boundary of the vertebral canal were defined according to the cavity. The front of the $\mathrm{CSA}_{\mathrm{vc}}$ is at the tail of intervertebral body or disc; the back of the $\mathrm{CSA}_{\mathrm{vc}}$ is at the front of ligamenta flava; the bilateral sides of the $\mathrm{CAC}_{\mathrm{vc}}$ are located at the wall of the pedicle of the vertebral arch. If the shapes of 2 sides on the vertebral canal image are open, the outer margins of the nerve root are used to locate the boundaries (Fig. 1). The mean CSA of the epidural space $\left(\mathrm{CSA}_{\mathrm{es}}\right)$, the mean CSA of epidural fibrosis $\left(\mathrm{CSA}_{\mathrm{ef}}\right)$, and the epidural fibrosis index were calculated according to the following formula:

$$
\begin{aligned}
& \text { mean } \mathrm{CSA}_{\mathrm{es}}=\Sigma\left(\mathrm{CSA}_{\mathrm{es}}-\mathrm{CSA}_{\mathrm{ds}}-\mathrm{CSA}_{\mathrm{nr}}\right) \times 1 / 5 ; \\
& \text { mean } \mathrm{CSA}_{\mathrm{ef}}=\Sigma\left[\left(\mathrm{CSA}_{\mathrm{es}}-\mathrm{CSA}_{\mathrm{ds}}-\mathrm{CSA}_{\mathrm{nr}}\right)\right] \times \text { the scale of a } \\
& \text { single slice } \times 10 \%] \times 1 / 5 ; \text { and } \\
& \quad \text { epidural fibrosis index }=\text { mean } \mathrm{CSA}_{\mathrm{ef}} / \text { mean } \mathrm{CSA}_{\mathrm{es}} \times 100 \%
\end{aligned}
$$

\section{Statistical Analysis}

All continuous data are presented as mean \pm SD and all categorical data as a percentage or number. Statistical analyses for comparisons between groups were performed using the unpaired Student t-test, chi-square test, and nonparametric Kruskal-Wallis test. Descriptive analysis and an independent samples test were also used. Statistical significance was set as $\mathrm{p}<0.05$. Intraclass correlation coefficient statistics were used to assess intrarater reliability when using the modified grading system; intraclass correlation coefficient values greater than 0.75 indicate excellent agreement. ${ }^{7}$ The power analysis of the statistical data was performed using SPSS computer software version 13.0.

\section{Results}

\section{Patient Characteristics}

Between March 2010 and December 2010, 75 patients were enrolled in the study: 37 patients were randomized to an MMC group and 38 to a control group. The distributions of patients' preoperative characteristics are shown for the evaluable patients in each group $(p>0.05)$ (Table 1). Thirteen of the 75 patients who underwent MED did not complete the follow-up. The drop-out rate is $17.33 \%$ (lower than 20\%).

\section{Clinical Follow-Up Results}

We observed no statistical significance between the MMC group and saline group regarding the results of neurological examinations and LSOQ scores at each interval. The results of the LSOQ scores and neurological examinations are shown in Table 2. Significant differences were determined ( $p<0.05$, t-test for paired samples) between preoperative LSOQ scores/neurological examinations and postoperative LSOQ scores/neurological examinations in both groups.

\section{Radiological Follow-Up Results}

Analyses of the MR images using the Ross grading system and modified grading system, including interpretations of enhanced and nonenhanced abnormalities, showed significantly different results between the MMC and control groups ( $\mathrm{p}<0.05$ ). Ross Grade 3 and 4 scars were observed more frequently in the MMC group $(24.7 \%)$ than in the control group $(12.2 \%$; see results in

TABLE 1: Baseline characteristics in 62 patients*

\begin{tabular}{lccc}
\hline & \multicolumn{2}{c}{ Group } & \\
\cline { 2 - 4 } Characteristic & MMC & Control & p Value \\
\hline mean age (yrs) & $35.67 \pm 10.66$ & $35.00 \pm 9.95$ & 0.813 \\
sex & 9 & 10 & 0.657 \\
$\quad$ female & 23 & 20 & 1.000 \\
male & $170.07 \pm 7.13$ & $170.07 \pm 6.39$ & 0.386 \\
mean height (cm) & $65.96 \pm 10.95$ & $68.89 \pm 13.51$ & 0.714 \\
mean weight (kg) & $48.89 \pm 41.69$ & $44.67 \pm 42.60$ & 0.213 \\
mean days of symptom aggravation & & & 15 \\
operative level & 11 & 15 & 0.450 \\
L4-5 & 21 & & 16 \\
$\quad$ L5-S1 & 14 & 14 & 0.735 \\
type of disc pathology & 18 & $0.3639 \pm 0.1134$ & 0.736 \\
$\quad$ extrusion & & $37.78 \pm 11.29$ & 0.121 \\
sequestration & $0.3544 \pm 0.0913$ & $39.63 \pm 19.61$ & \\
mean nucleus protrusion rate & $38.70 \pm 8.61$ & & \\
mean op time (mins) & $49.07 \pm 24.22$ & & \\
mean intraop bleeding (ml) &
\end{tabular}

* Mean values are presented \pm SD. 
Inhibition of epidural fibrosis by mitomycin C

TABLE 2: Clinical follow-up results

\begin{tabular}{|c|c|c|c|}
\hline \multirow[b]{2}{*}{ Factor } & \multicolumn{2}{|c|}{ Group } & \multirow[b]{2}{*}{ p Value } \\
\hline & MMC & Control & \\
\hline \multicolumn{4}{|c|}{ low-back pain \& leg pain severity scores } \\
\hline baseline & $44.1975 \pm 15.3023$ & $46.1420 \pm 15.3714$ & 0.643 \\
\hline \multicolumn{4}{|l|}{7 days } \\
\hline actual value & $9.7839 \pm 3.9901$ & $9.2284 \pm 6.1059$ & 0.694 \\
\hline change from baseline & $34.4136 \pm 15.5446$ & $36.9136 \pm 15.3725$ & 0.555 \\
\hline \multicolumn{4}{|l|}{$3 \mathrm{mos}$} \\
\hline actual value & $5.4012 \pm 4.8003$ & $4.6296 \pm 4.0187$ & 0.525 \\
\hline change from baseline & $38.7963 \pm 16.9248$ & $41.5123 \pm 16.5219$ & 0.553 \\
\hline \multicolumn{4}{|l|}{$6 \mathrm{mos}$} \\
\hline actual value & $1.6975 \pm 2.2347$ & $1.0185 \pm 1.0163$ & 0.157 \\
\hline change from baseline & $42.5000 \pm 15.2262$ & $45.1235 \pm 15.2607$ & 0.530 \\
\hline \multicolumn{4}{|c|}{ functional disability w/ activity-related pain severity scores } \\
\hline baseline & $73.3333 \pm 11.9099$ & $74.0741 \pm 10.7343$ & 0.811 \\
\hline \multicolumn{4}{|l|}{$3 \mathrm{mos}$} \\
\hline actual value & $52.7407 \pm 6.8253$ & $52.8519 \pm 6.2984$ & 0.951 \\
\hline change from baseline & $20.5926 \pm 14.8874$ & $21.2222 \pm 12.9536$ & 0.869 \\
\hline \multicolumn{4}{|l|}{$6 \mathrm{mos}$} \\
\hline actual value & $47.6296 \pm 1.8218$ & $48.7407 \pm 6.6018$ & 0.403 \\
\hline change from baseline & $25.7037 \pm 12.0439$ & $25.3333 \pm 11.5360$ & 0.909 \\
\hline \multicolumn{4}{|l|}{ physical symptoms severity scores } \\
\hline baseline & $40.2963 \pm 10.5787$ & $39.8518 \pm 12.1772$ & 0.887 \\
\hline \multicolumn{4}{|l|}{7 days } \\
\hline actual value & $32.1481 \pm 7.4818$ & $29.9259 \pm 6.5979$ & 0.252 \\
\hline change from baseline & $8.1481 \pm 11.7136$ & $9.9259 \pm 11.0590$ & 0.569 \\
\hline \multicolumn{4}{|l|}{$3 \mathrm{mos}$} \\
\hline actual value & $23.4074 \pm 4.9245$ & $24.4444 \pm 5.0026$ & 0.446 \\
\hline change from baseline & $16.8889 \pm 11.4936$ & $15.4074 \pm 11.4598$ & 0.637 \\
\hline \multicolumn{4}{|l|}{$6 \mathrm{mos}$} \\
\hline actual value & $20.5926 \pm 1.4481$ & $21.0370 \pm 1.7830$ & 0.320 \\
\hline change from baseline & $19.7037 \pm 11.0343$ & $18.8148 \pm 12.1435$ & 0.779 \\
\hline
\end{tabular}

Table 3). Results stratified by the modified grading system are showed in Table 4. Intrarater agreement was "excellent" for the 2 orthopedists. Intraclass correlation coefficient values were as follows: for average $\mathrm{CSA}_{\mathrm{es}}, 0.909$ (95\% CI 0.849-0.948); for average $\mathrm{CSA}_{\mathrm{ef}}, 0.885$ (95\% CI $0.812-0.934)$; and for epidural fibrosis index, 0.912 (95\% CI 0.855-0.950). We also found significant differences in the average CSA of the epidural scar and epidural fibrosis index between the MMC and control groups ( $<0.05$ ).

\section{Safety}

No adverse effects such as wound infections, wound healing delay, dural tear, spinal epidural hematoma, or arachnoiditis were observed as the result of MMC usage and the surgical procedure. Mean duration of hospital stay was 13 days. Although 1 recurrent disc herniation on follow-up MR images was observed in the MMC group and control group, there was no evidence of a significant and consistent relationship between recurrent disc hernia- tion and the applied MMC. Both patients had uneventful postoperative recoveries and did not require a second operation.

\section{Discussion}

Although epidural fibrosis is a natural consequence of discectomy to replace the normal epidural fat with fibrotic tissue and to bind the dural sac and nerve roots to the surrounding structures, excessive scarring or scarring in regions where adhesions cause anatomical and electrophysiological dysfunction may contribute to undesirable postoperative symptoms and pain recurrence. ${ }^{2,8,11}$ Anterior and posterior adhesions of the dura and nerve roots to epidural fibrosis tissues near spine surgery sites are known to cause intractable pain, which cannot be easily relieved by an analgesic agent or by physical therapy programs. Additionally, epidural fibrosis increases the risks of dural tears and nerve roots injury and prolongs operat- 
TABLE 3: Radiological scores using the Ross grading system*

\begin{tabular}{cccc}
\hline & & \multicolumn{3}{c}{ Group } & \\
\cline { 3 - 4 } Evaluator & Grade & MMC $(\mathrm{n}=600)$ & Control $(\mathrm{n}=640)$ \\
& 0 & 0.6781 & 0.6017 \\
& 1 & 0.1000 & 0.0867 \\
& 2 & 0.0672 & 0.0717 \\
& 3 & 0.0656 & 0.1133 \\
& 4 & 0.0891 & 0.1267 \\
& 0 & 0.6641 & 0.6150 \\
& 1 & 0.0922 & 0.0700 \\
& 2 & 0.0750 & 0.0783 \\
& 3 & 0.0797 & 0.1117 \\
& 4 & 0.0891 & 0.1250 \\
\hline
\end{tabular}

\footnotetext{
* Nonparametric Kruskal-Wallis test was used to compare radiological scores between MMC and control groups.

† Evaluator 1: Radiological scores between MMC group and control group were statistically significant.

‡ Evaluator 2: Radiological scores between MMC group and control group were statistically significant.
}

ing time in the second-time surgery. Given these factors, presenting an effective method for preventing epidural fibrosis has assumed more pressing importance. Various materials and drugs have been used to prevent or minimize epidural fibrosis around neural structures, including fat graft, absorbable gelatin sponge, steroids, polylactic acid, polymethylmethacrylate, carboxymethyl cellulose, low-dose radiation, heparinized material, ADCON-L, urokinase, MMC, tissue plasminogen activator, and so on. ${ }^{5,9,10,14}$ Some of these treatments have been found to prevent fibrosis successfully after discectomy in animal models. Many clinical trials also have been conducted but have mostly ended with frustrating results.

Mitomycin C, an ankylosing antibiotic substance, can be easily and rapidly absorbed into cells to inhibit RNA and protein synthesis as well as DNA replication. As a potential suppressor of fibroblast proliferation and differentiation, mitomycin $\mathrm{C}$ has been used topically in ophthalmological and urological surgery to suppress fibrotic enlargement at various concentrations of 0.1 and $0.5 \mathrm{mg}$ / $\mathrm{ml}$ for 2 and 5 minutes, respectively. ${ }^{16,18}$ This inhibition of epidural fibrosis after lumbar disc surgery also has been observed in experimental studies of laminectomy models. Celik and colleagues ${ }^{4}$ performed a prospective randomized clinical study to validate the antifibrotic effect in lumbar disc surgery. However, no benefits were observed clinically or radiographically. Lack of better measures of peridural fibrosis and limited intervention efforts of
MMC may account for the negative result. Based on previous clinical studies, we tried to produce the maximal drug effect by using a piece of cotton wool impregnated with $0.5 \mathrm{mg} / \mathrm{ml} \mathrm{MMC}$ around the nerve, including the anterior, lateral, posterior space. Additionally, we used 2 different grading systems to evaluate the MR images.

We found a significant difference in the extent of epidural fibrosis when comparing the MMC group and the saline group. Epidural fibrosis index was $0.2058 \pm 0.7672$ in the MMC group and $0.2542 \pm 0.0728$ in the saline group. This result shows that MMC could effectively reduce epidural fibrosis and prevent dural adhesions radiologically. Additionally, analyses failed to show a satisfactory clinical difference for MMC. We believe that 2 possibilities may explain the incongruity between the radiological result and clinical outcome: First, flavectomy was performed using a ligamentum flavum longitudinal splitting in the MED procedure, and this helped to retain the ligamentum flavum maximally. The ligamentum flavum was considered to be the natural barrier against epidural scarring. ${ }^{12}$ Second, compared with total or semilaminectomy, the limited dural exposure in the MED contributes to unremarkable postoperative persistent pain, which could be partially explained by epidural scar. The clinical effects of MMC may be better determined in procedures that involve substantially greater surgical exposure. Several authors have studied the relationship between epidural fibrosis and recurrent pain, and they came to the conclusion that epidural fibrosis

TABLE 4: Radiological scores using the modified grading system

\begin{tabular}{cccc}
\hline & \multicolumn{2}{c}{ Group } & \\
\cline { 2 - 3 } Parameter & MMC & Control & V Value \\
\hline${\text { mean } \mathrm{CAS}_{\mathrm{ds}}\left(\mathrm{mm}^{2}\right)}^{\text {mean } \mathrm{CAS}_{\text {es }}\left(\mathrm{mm}^{2}\right)}$ & $183.0778 \pm 54.157$ & $185.6074 \pm 46.5535$ & 0.855 \\
epidural fibrosis index & $36.1444 \pm 14.1638$ & $45.9407 \pm 14.1561$ & $0.014^{*}$ \\
\hline${ }^{*}$ Mean CAS $_{\text {es }}$ compared between MMC group and control group $(\mathrm{p}<0.05)$. & $0.021 \dagger$ \\
$\dagger$ Epidural fibrosis index compared between MMC group and control group $(\mathrm{p}<0.05)$. &
\end{tabular}


should be considered a radiological finding independent of the clinical outcome. ${ }^{1}$

Mitomycin $\mathrm{C}$ has an excellent safety profile with no medically significant adverse events or complications attributed to it at lower concentration. All surgical areas were rinsed well to eliminate the surplus MMC. No delayed healing of the surgical incision was observed; the tubular retractor was considered to be protective because it prevented MMC from touching the surgical incision. Otherwise, MMC is still not recommended if the dural tear occurs during the operation.

\section{Conclusions}

The preliminary results of this prospective, doubleblinded, randomized clinical trial have demonstrated that topical treatment with MMC in a concentration of 0.5 $\mathrm{mg} / \mathrm{ml}$ is effective for inhibiting postoperative epidural fibrosis after MED. Multicenter clinical trials should be conducted to validate the efficacy of MMC in total or semilaminectomy.

\section{Disclosure}

The authors report no conflict of interest concerning the materials or methods used in this study or the finding specified in this paper.

This work was supported by the National Natural Science Foundation of China (Grant Nos. 30973058 and 81171694); the Program for Development of Innovative Research Team in the First Affiliated Hospital of NJMU (No. IRT-015); and a project funded by the Priority Academic Program Development of Jiangsu Higher Education Institutions.

Author contributions to the study and manuscript preparation include the following. Conception and design: $\mathrm{Cao}, \mathrm{Liu}, \mathrm{Wu}$. Acquisition of data: Liu, Hong, Wu. Analysis and interpretation of data: Cao, Liu, Sui. Drafting the article: Sui. Critically revising the article: all authors. Reviewed submitted version of manuscript: all authors. Approved the final version of the manuscript on behalf of all authors: Cao. Statistical analysis: Cao, Liu, Sui. Administrative/technical/ material support: Cao, Liu, Sui, Wu. Study supervision: Wu.

\section{Acknowledgments}

The authors thank Suyang Zhuang, Shaodong Zhang, Jun Wang, Chen Meng, and Xiaohu Wang for their help in MRI evaluation.

\section{References}

1. Almeida DB, Prandini MN, Awamura Y, Vitola ML, Simião MP, Milano JB, et al: Outcome following lumbar disc surgery: the role of fibrosis. Acta Neurochir (Wien) 150:1167-1176, 2008

2. BenDebba M, Augustus van Alphen H, Long DM: Association between peridural scar and activity-related pain after lumbar discectomy. Neurol Res 21 (Suppl 1):S37-S42, 1999

3. Bendebba M, Dizerega GS, Long DM: The Lumbar Spine Outcomes Questionnaire: its development and psychometric properties. Spine J 7:118-132, 2007

4. Celik SE, Altan T, Celik S, Göksu K, Ince I, Kapran Z: Mito- mycin protection of peridural fibrosis in lumbar disc surgery. J Neurosurg Spine 9:243-248, 2008

5. Cemil B, Tun K, Kaptanoglu E, Kaymaz F, Cevirgen B, Comert A, et al: Use of pimecrolimus to prevent epidural fibrosis in a postlaminectomy rat model. Laboratory investigation. J Neurosurg Spine 11:758-763, 2009

6. Danielson BI, Willén J, Gaulitz A, Niklason T, Hansson TH: Axial loading of the spine during CT and MR in patients with suspected lumbar spinal stenosis. Acta Radiol 39:604-611, 1998

7. Fleiss JL: The measurement of inrater agreement, in: Statistical Methods for Rates and Proportions, ed 2. New York: John Wiley \& Sons, 1981, pp 211-236

8. Jou IM, Tai TW, Tsai CL, Tsai TM, Yung WS, Jung YC: Spinal somatosensory evoked potential to evaluate neurophysiologic changes associated with postlaminotomy fibrosis: an experimental study. Spine (Phila Pa 1976) 32:2111-2118, 2007

9. Kasimcan MO, Bakar B, Aktaş S, Alhan A, Yilmaz M: Effectiveness of the biophysical barriers on the peridural fibrosis of a postlaminectomy rat model: an experimental research. Injury 42:778-781, 2011

10. Liu J, Ni B, Zhu L, Yang J, Cao X, Zhou W: Mitomycin C-polyethylene glycol controlled-release film inhibits collagen secretion and induces apoptosis of fibroblasts in the early wound of a postlaminectomy rat model. Spine J 10:441-447, 2010

11. Maroon JC, Abla A, Bost J: Association between peridural scar and persistent low back pain after lumbar discectomy. Neurol Res 21 (Suppl 1):S43-S46, 1999

12. Ozer AF, Oktenoglu T, Sasani M, Bozkus H, Canbulat N, Karaarslan E, et al: Preserving the ligamentum flavum in lumbar discectomy: a new technique that prevents scar tissue formation in the first 6 months postsurgery. Neurosurgery 59 (1 Suppl 1):ONS126-ONS133, 2006

13. Rabb CH: Failed back syndrome and epidural fibrosis. Spine J 10:454-455, 2010

14. Rönnberg K, Lind B, Zoega B, Gadeholt-Göthlin G, Halldin $\mathrm{K}$, Gellerstedt M, et al: Peridural scar and its relation to clinical outcome: a randomised study on surgically treated lumbar disc herniation patients. Eur Spine J 17:1714-1720, 2008

15. Ross JS, Obuchowski N, Modic MT: MR evaluation of epidural fibrosis: proposed grading system with intra- and inter-observer variability. Neurol Res 21 (1 Suppl 1):S23-S26, 1999

16. Su C, Yao C, Lu S, Zhang A, Cao X, Teng G, et al: Study on the optimal concentration of topical mitomycin-C in preventing postlaminectomy epidural adhesion. Eur J Pharmacol 640: 63-67, 2010

17. Wu X, Zhuang S, Mao Z, Chen H: Microendoscopic discectomy for lumbar disc herniation: surgical technique and outcome in 873 consecutive cases. Spine (Phila Pa 1976) 31:2689-2694, 2006

18. Yildiz KH, Gezen F, Is M, Cukur S, Dosoglu M: Mitomycin C, 5-fluorouracil, and cyclosporin A prevent epidural fibrosis in an experimental laminectomy model. Eur Spine J 16:15251530,2007

Manuscript submitted June 5, 2012.

Accepted January 31, 2013.

Please include this information when citing this paper: published online March 8, 2013; DOI: 10.3171/2013.1.SPINE12564.

Address correspondence to: Xiaojian Cao, M.D., Ph.D., 300 Guangzhou Road, Nanjing 210029, Jiangsu Province, China. email: xiaojiancao@gmail.com. 